Check for updates

Cite this: RSC Adv., 2019, 9, 14677

\title{
Wavelength-dependent photoconductivity of single-walled carbon nanotube layers $\uparrow$
}

\author{
Serguei Smirnov, (D) a llya V. Anoshkin, (D) ${ }^{\text {b }}$ Andrey Generalov, (D) \\ Dmitri V. Lioubtchenko (D) *ad and Joachim Oberhammer (D) ${ }^{a}$
}

\begin{abstract}
A number of electronic devices such as phase shifters, polarizers, modulators, and power splitters are based on tunable materials. These materials often do not meet all the requirements namely low losses, fast response time, and technological compatibility. Novel nanomaterials, such as single-walled carbon nanotubes, are therefore widely studied to fill this technological gap. Here we show how the dielectric constant of single-walled carbon nanotube layers can be substantially modified by illuminating them due to unique light-matter interactions. We relate the optical excitation of the nanotube layers to the illumination wavelength and intensity, by resistance and capacitance measurements. The dielectric constant is modified under laser illumination due to the change of material polarization and free carrier generation, and is shown to not be temperature-related. The findings indicate that SWCNT layers are a prospective tunable optoelectronic material for both high and low frequency applications.
\end{abstract}

Received 26th February 2019

Accepted 30th April 2019

DOI: 10.1039/c9ra01467e

rsc.li/rsc-advances visible, and IR spectra that are attributed to electronic transitions between energy states of semiconducting and metallic SWCNTs. ${ }^{7}$ These unique absorption properties exited the interest in nanotubes as optoelectronic material, leading to diverse studies such as applications in solar cells; ${ }^{8,9}$ photoluminescence for imaging and sensing; ${ }^{10,11}$ high optical nonlinearity with fast response for optical communication systems; ${ }^{12,13}$ optical transitions in SWCNT fieldeffect transistors with electrostatic gating control; ${ }^{14-16}$ photoinduced molecular desorption for gas sensing; ${ }^{17,18}$ and terahertz wave generation and detection for flexible and wearable electronics. ${ }^{19}$

Whereas most recent studies of light-matter interactions in SWCNTs are reported on separated nanotubes, the samples require complex processing steps such as centrifugation in liquid solution. ${ }^{\mathbf{2 0 , 2 1}}$ Instead, thin SWCNT layers are of interest due to the simple method of dry transfer. ${ }^{22,23}$ The technique allows an easy transfer of as-fabricated layers onto a wide variety of substrates with possibility of large-scale applications in tunable electromagnetic wave reflecting and absorbing surfaces. Integration of SWCNT layers has already been successfully demonstrated in micro- and nanoelectromechanical systems and sensors. ${ }^{23,24}$ A typical layer is naturally composed of randomly-oriented nanotubes with a proportion of approximately $1 / 3$ metallic to $2 / 3$ semiconducting types. ${ }^{25}$ The absorption properties of bundled SWCNTs are characterized by fast relaxation $(<1 \mathrm{ps})$ with intertube energy transfer from semiconducting to metallic nanotubes, followed by rapid nonradiative cooling. ${ }^{26,27}$ The electrical responses of isolated nanotubes ${ }^{28,29}$ and SWCNT layers $^{30,31}$ to light excitation have been studied in the mid- and far-IR regions. The results indicate a generation of stronglybound excitons and an enhancement of the IR absorption. 
DC-level measurements have been reported as a light-induced photocurrent in individual nanotubes ${ }^{32,33}$ as well as in SWCNT layers. ${ }^{34,35}$ Measurement conditions such as contact resistance between electrodes and SWCNTs, atmospheric conditions, and light intensity have been shown to significantly impact the results.

However, the illumination wavelength dependence is not understood completely. Peaks in the photoresponse were observed in the mid-IR range, related to the first and second electronic transitions in the semiconducting nanotubes. ${ }^{32,35}$ The molecular photodesorption rate was shown to be wavelengthdependent in the visible and near-UV range. ${ }^{17} \mathrm{~A}$ change in the photoconductivity was observed in double-walled CNTs as a function of illumination wavelength, explained by an excitation of surface plasmons. ${ }^{36}$ Due to the heterogeneous nature of the nanotube layers, identifying light absorption mechanisms is difficult as several physical processes occur simultaneously.

Previously, we reported an experimental tuning of the dielectric constant of thin SWCNT layers in the frequency range of 75 $\mathrm{GHz}$ to $1 \mathrm{THz}$ under light illumination. ${ }^{23}$ In this paper, we relate the illumination wavelength and intensity to the optical excitation of nanotube layers, with the perspective of their application as tunable optoelectronic material. We measure the resistance and capacitance of the samples under laser illumination in the wavelength range of $488 \mathrm{~nm}$ to $785 \mathrm{~nm}$. We distinguish a slow and a fast regime that are wavelength-dependent. The dielectric constant is tuned due to free charge carrier generation and due to the change of material polarization, like ferroelectric and liquid crystals. However, the main advantage of carbon nanotubes is that these processes are much faster.

\section{Experimental}

Experimental investigations were performed on SWCNT layers synthesized by aerosol chemical vapor deposition, ${ }^{37}$ with an optical transparency of $80 \%$, measured at $550 \mathrm{~nm}$. The randomlyoriented SWCNT network and the bundling of individual nanotubes is shown in Fig. 1. Scanning and transmission electron microscopy was performed with a high-resolution scanning electron microscope JEOL JSM-7500FA at $2 \mathrm{kV}$ and a spherical aberration corrected transmission electron microscope JEOL
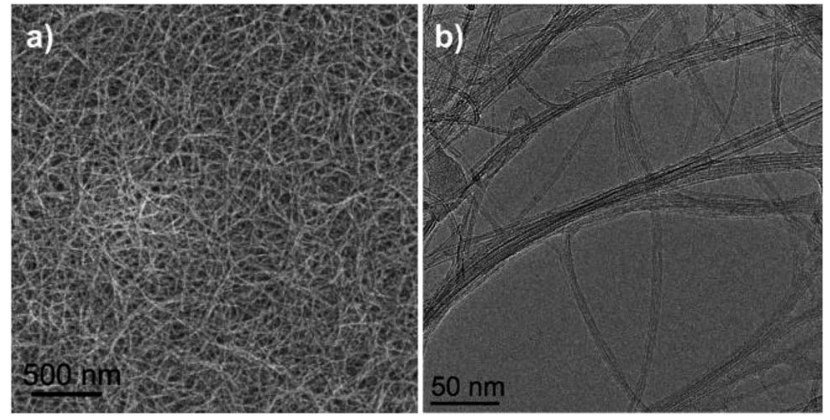

Fig. 1 (a) Scanning electron microscopy image of the randomlyoriented SWCNTs network. (b) Transmission electron microscopy image of the bundling of individual nanotubes.
JEM-2200FS at $80 \mathrm{kV}$. An average nanotube length of $5 \mu \mathrm{m}$ to 15 $\mu \mathrm{m}$ was determined by visual inspection..$^{\mathbf{3 8}, 39}$

The optical absorbance $(A)$ of the SWCNT layers was derived from transmission $(T)$ measurements, obtained with a PerkinElmer 950 UV-vis-NIR spectrometer in the range of 200$2500 \mathrm{~nm}$ as: $A=\log _{10}(100 / T)$ (see Fig. 2a). ${ }^{40}$ The spectrum was measured with a double beam spectrometer for a solid SWCNT layer on a quartz substrate without liquid treatment. The presence of the strong peak at $280 \mathrm{~nm}$ corresponds to the $\pi$-plasmon with a long tail up to the IR range. The electronic transitions of the SWCNTs (marked as $\mathrm{S}_{11}, \mathrm{~S}_{22}$, and $\mathrm{M}_{11}$ ) are overlapping with the tail of the $\pi$-plasmon. ${ }^{\mathbf{4 1 , 4 2}}$ According to the

a)

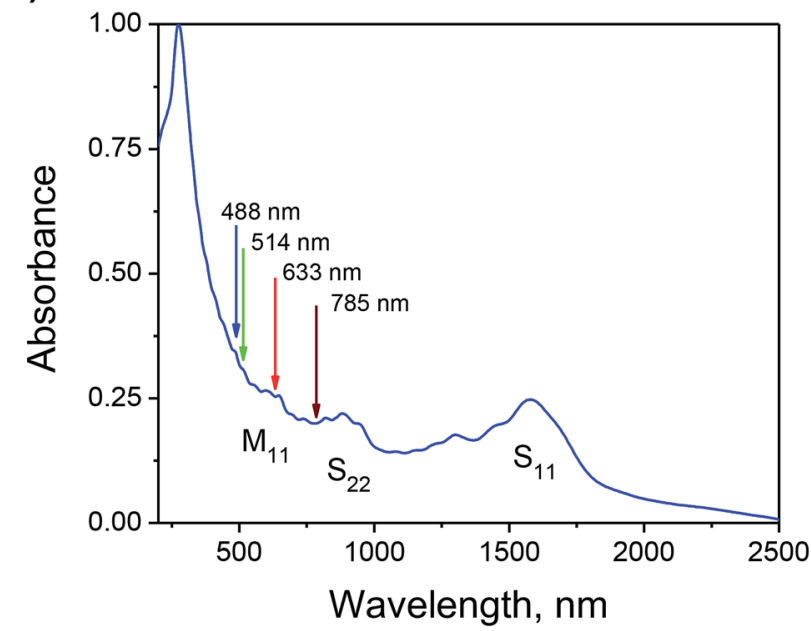

b)

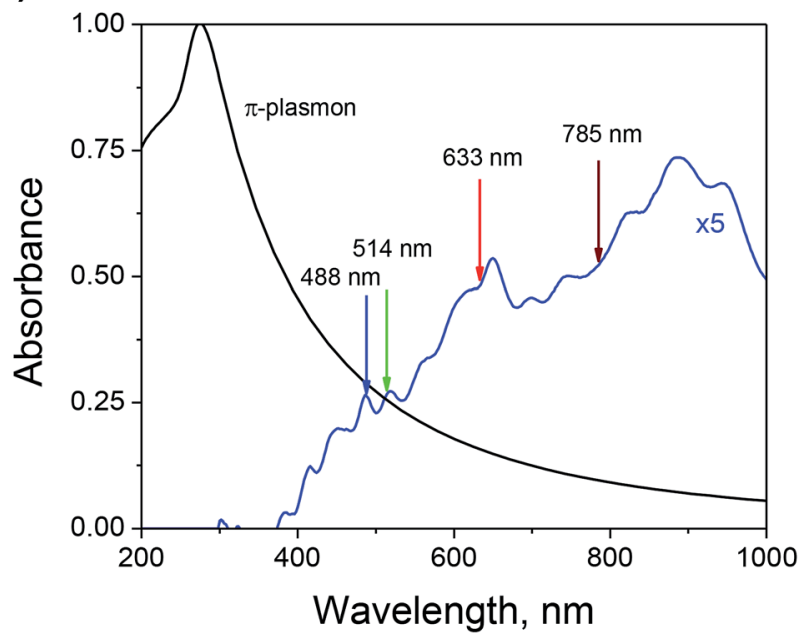

Fig. 2 Optical absorbance spectrum of the SWCNT layer. (a) The average nanotube diameter was determined from the position of the absorbance peak of the first electronic transition $\mathrm{S}_{11}$ in semiconducting SWCNTs. The second electronic transition in semiconducting nanotubes $S_{22}$ and the first one in metallic nanotubes $M_{11}$ are indicated for reference. The arrows indicate the illuminating laser wavelengths. (b) Decomposition of the optical absorbance spectrum of the SWCNT layer. The $\pi$-plasmon peak was approximated by a non-linear fit. The peaks due to electronic transitions in the nanotubes were obtained by subtracting the $\pi$-plasmon peak from the total absorbance; their amplitude was multiplied by 5 for visibility. 
position of the main absorbance peak of the first electronic transition in the semiconducting tubes $\left(\mathrm{S}_{11}\right)$ at $1600 \mathrm{~nm}$, an average nanotube diameter of $1.26 \mathrm{~nm}$ was determined with the Weisman plot (see Fig. S1 and Table S1 $\uparrow$ for additional information). ${ }^{43,44}$ We decompose the absorbance spectrum as shown in Fig. $2 \mathrm{~b}$. The $\pi$-plasmon peak was approximated by a nonlinear fit. ${ }^{45}$ The peak was then subtracted from the total absorbance to obtain the contribution of the electronic transitions (see additional information in ESI, Fig. S2†).

A Horiba Jobin-Yvon Labram HR Raman was used for the illumination of SWCNT samples and Raman spectrum measurements (see ESI, Fig. S3 $\dagger$ ). Four lasers were used: two argon lasers with wavelengths of $488 \mathrm{~nm}$ and $514 \mathrm{~nm}$, a heliumneon laser with a wavelength of $633 \mathrm{~nm}$, and an infrared diode laser with a wavelength of $785 \mathrm{~nm}$. The lasers were controlled with build-in attenuators to achieve the power levels given in Table S2. $\uparrow$ The Raman spectra were collected at $633 \mathrm{~nm}$ with a $50 \times$ objective, 0.75 numerical aperture, $600 \mathrm{Gr} \mathrm{mm}^{-1}$ spectral grating, and $3 \mathrm{~dB}$ attenuation resulting in $8.5 \mathrm{~mW}$ incident power. The signal integration time was $10 \mathrm{~s}$. The measurements were performed on up to 10 positions per sample, without noticeable difference. The Raman spectrum of the sample is given in Fig. S4, $\dagger$ indicating a low proportion of nontubular impurities by the low D/G peaks ratio of 0.04 . The RBM region of the Raman spectrum was decomposed and fitted with Lorentzian curves (see Fig. S5 and fit parameters in Table S3†). From the RBM peak positions, diameters of nanotubes in the sample are determined to range from $0.9 \mathrm{~nm}$ to $2.0 \mathrm{~nm}$.

Two-point impedance (capacitance and resistance) measurements were performed with an Agilent B1500A semiconductor device analyzer at $1 \mathrm{MHz}$. Parallel metal contacts were used with a $3 \mathrm{~mm}$ spacing. Four-point resistance measurements were performed with an Agilent 34401A digital multimeter. X-shaped gold microcontacts were used with a separation of $10 \mu \mathrm{m}$ between central electrodes (see Fig. 3a and S3†).

\section{Results and discussion}

\subsection{Resistance measurements}

The SWCNT samples were transferred from nitrocellulose filter onto glass substrates with the electrodes by dry transfer (see ESI, Fig. S6 $\dagger$ ). The illumination beam was focused to a spot with an approximate diameter of $7 \mu \mathrm{m}$ for all lasers to exclude the influence of the metal-SWCNT barrier effect (see ESI, Fig. S7†). Complete four-point resistance measurement results are provided in Fig. S8. $\dagger$ A comparison of the change in resistance under illumination as a function of time is shown in Fig. $3 \mathrm{~b}$ for wavelengths of $488 \mathrm{~nm}, 514 \mathrm{~nm}, 633 \mathrm{~nm}$, and $785 \mathrm{~nm}$, and comparable laser powers. Two distinct behaviors can be observed, suggesting different mechanisms causing the change in resistance. For the wavelengths of $488 \mathrm{~nm}$ and $514 \mathrm{~nm}$, the resistance is characterized by a slow exponentially-decaying increase with the illumination time (ON-state), and by a corresponding slow decrease during the OFF-state (see additional details in ESI, Fig. S9†). For the wavelengths of $633 \mathrm{~nm}$ and $785 \mathrm{~nm}$, the illumination is accompanied by a fast jump of the resistance, followed by a slow increase. And respectively, the OFF-state is characterized by a fast drop in the resistance, followed by a slow decrease. A comparison of the resistance change as a function of the laser power and wavelength is shown in Fig. 3c, where the two behaviors are recognized.

We attribute the long-term drifts of the resistance to a slow heating of the SWCNT network. We distinguish the slow exponential variations of the resistance, observed at all wavelengths, from the fast responses to illumination at $633 \mathrm{~nm}$ and $785 \mathrm{~nm}$. We explain the difference in behavior with the help of the decomposed absorbance spectrum from Fig. 2b. At shorter wavelengths ( $488 \mathrm{~nm}$ and $514 \mathrm{~nm}$ ), the absorbance is dominated by the $\pi$-plasmon peak. The resistance change is then limited by the slow dynamics of oxygen and water desorption, affecting the doping of the SWCNT network. ${ }^{46}$ At longer wavelengths ( $633 \mathrm{~nm}$ and $785 \mathrm{~nm}$ ), the total absorbance is smaller which results in lower resistance changes. However, absorbance peaks due to electronic transitions in SWCNTs become relevant and the fast jump of the resistance can be related to the generation of free charge carriers. ${ }^{47,48}$ The jump in resistance is also observed at shorter wavelengths but on a smaller scale.

\section{2. $C-V$ measurements}

Complete two-point impedance measurement results are provided in Fig. S10. $\dagger$ A comparison of the change in capacitance induced by the illumination is shown in Fig. $3 \mathrm{~d}$ as a function of time for the four wavelengths and similar power levels. The capacitance change is close to the "noise level" of the measurement setup and required post-processing. Nevertheless, the results can be compared qualitatively. In contrast to the resistance, the capacitance is decreasing during illumination, and returning to its initial value during the OFF-state. Fig. 3e summarizes the change in capacitance and resistance as a function of the illumination wavelength. For shorter wavelengths, larger variations of capacitance and resistance are observed that can be correlated to the stronger absorbance in that part of the spectrum (see Fig. 2a).

We explain the laser-induced capacitance change of the SWCNT layer by a polarization under light illumination. The polarization leads to a change of the dipole moment, resulting in the change of the dielectric constant of the nanotube layer. Moreover, the illumination causes the generation of free charge carriers and the resistance of the nanotube network is primarily defined by the hopping and tunnelling charge transport at the nanotube intersections and bundles. ${ }^{49,50}$ The resistance is exponentially proportional to the intertube distance, which we suppose increases due to the polarization. This results in an unexpected increase of resistance under illumination, which has been similarly observed in layers of double-walled carbon nanotubes. ${ }^{36}$ Additionally, the increase of the intertube distance results in a decrease of the quantum capacitance of the nanotubes, enhanced by the bundling. ${ }^{51}$ This direct control over the dielectric constant of thin SWCNT layers and nanotube composites makes them a potential candidate as tunable optoelectronic material. 


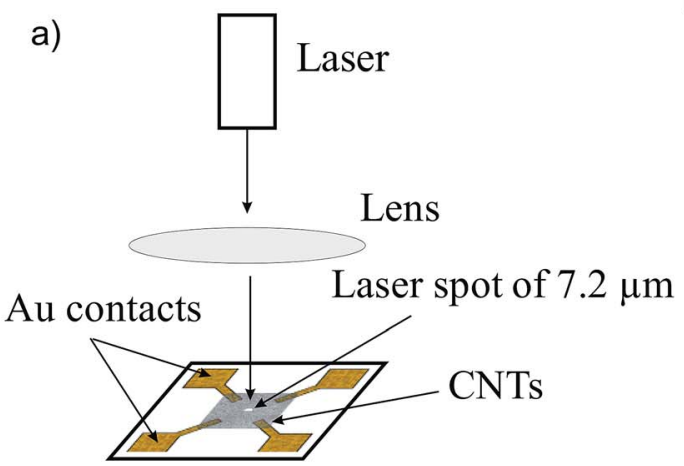

c)

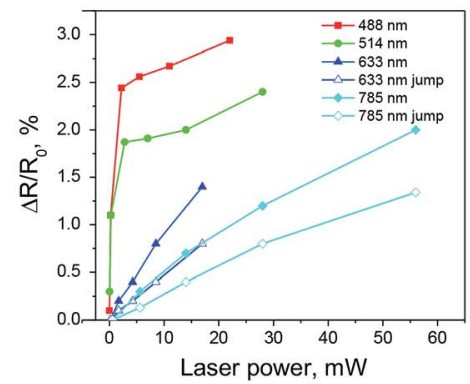

d)

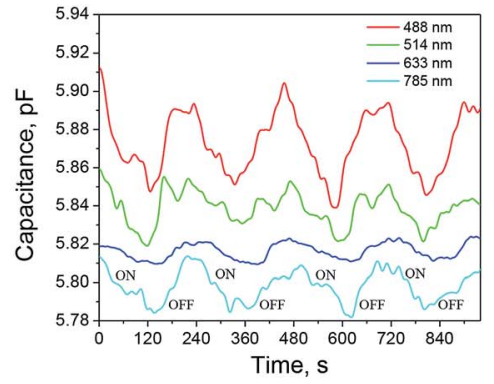

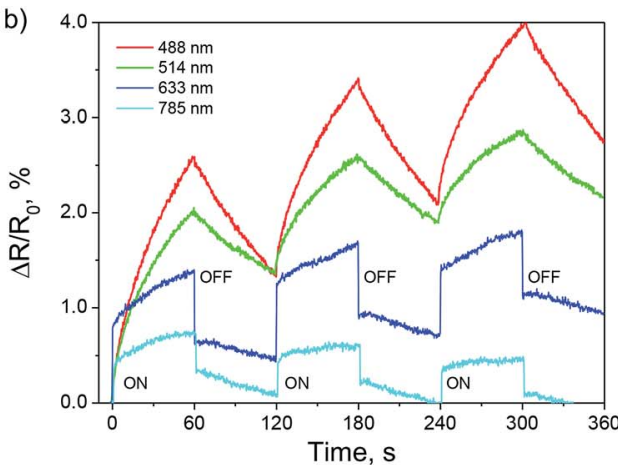

e)

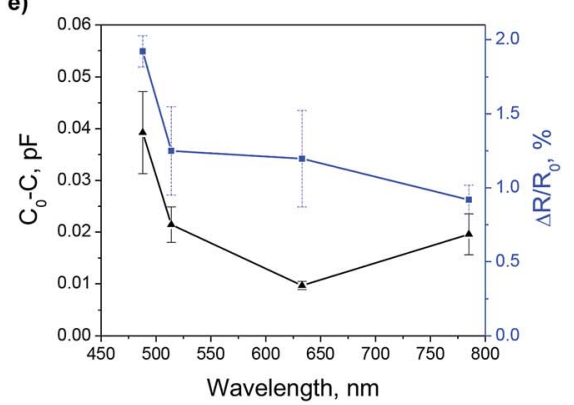

Fig. 3 Characterization of thin SWCNT layers by resistance and capacitance measurements. (a) Schematic drawing of the four-point resistance measurement setup. The SWCNT layer is transferred on a quartz substrate with gold contact electrodes. The sample is illuminated by a laser focused to a spot size of $7.2 \mu \mathrm{m}$. (b) Normalized change of the SWCNT layer's resistance during illumination with four wavelengths and similar power levels. The labels indicate one minute ON and OFF illumination cycles. (c) Normalized change of the SWCNT layer's resistance under illumination as a function of laser power and wavelength. (d) Change of the SWCNT layer's capacitance during illumination with four laser wavelengths and similar power levels. The labels indicate two minutes ON and OFF illumination cycles. (e) Comparison of the change in capacitance and normalized resistance of the SWCNT layer as a function of the illumination wavelength for similar power levels.

\subsection{Temperature dependence}

A potential explanation for the change of resistance and capacitance is the heating of the SWCNTs layer by the laser. To discard this effect, several experiments were carried out with Raman and optical spectroscopy. Raman spectra taken before and during prolonged (30 $\mathrm{min}$ ) laser illumination are shown in

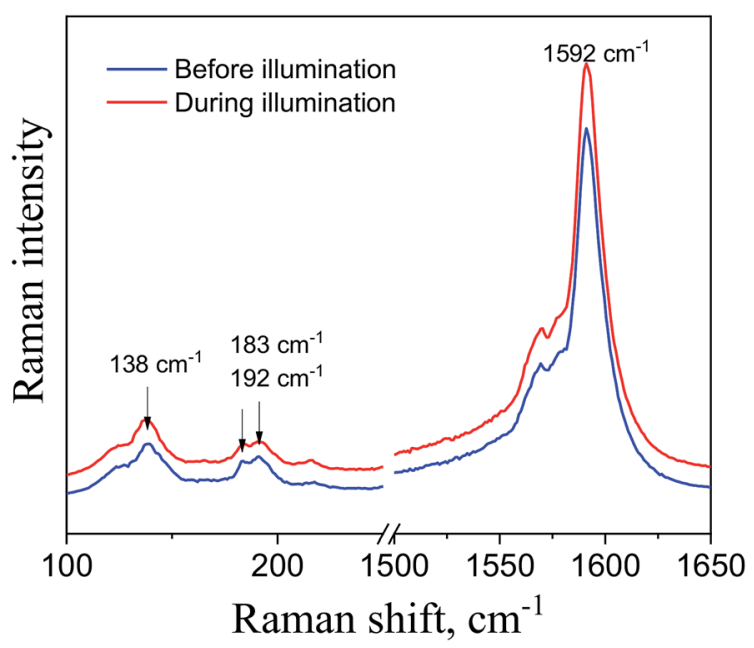

Fig. 4 Raman spectra before and during laser illumination of the SWCNT layer. The main peak positions (RBM and $\mathrm{G}^{+}$) are not shifted, indicating that no thermal heating of the sample occurs. The spectra are offset vertically for visibility.
Fig. 4. The positions of the RBM and G peaks are not shifted, which demonstrates that no excessive heating of the SWCNT network occurs. ${ }^{52}$ In comparison, the shift of peaks was observed with laser powers at least 5 times higher. ${ }^{53,54}$

The thermal influence on the optical absorption spectra of SWCNT layers was also studied in situ. The absorbance measured for a different sample (see ESI, Fig. S11†) shows that no changes occur due to heating up to $190{ }^{\circ} \mathrm{C}$ in the wavelength range of interest (488 $\mathrm{nm}$ to $785 \mathrm{~nm}$ ). Significant changes in the absorbance appear only in the infrared region (wavelengths above $1300 \mathrm{~nm}$ ). The shift is explained by the influence of oxygen and other oxidizing agents $\left(\mathrm{NO}_{2}\right.$ or $\left.\mathrm{SO}_{2}\right)$ adsorbed by the SWCNT surface, which induces surface p-doping and a modification of the SWCNT valence band structure. ${ }^{55,56}$ Consequently, the heating detaches the dopants, which leads to an increase of the absorbance around the $S_{11}$ transition of the semiconducting nanotubes. In our case, the illuminating laser wavelengths lie around the $\mathrm{M}_{11}$ and $\mathrm{S}_{22}$ transitions of the SWCNTs, where the absorbance is not impacted by temperature.

\section{Conclusions}

We have experimentally demonstrated the tuning of the dielectric properties of thin SWCNT layers by impedance measurements during laser illumination. Light is shown to decrease both the conductivity and the capacitance of the sample, without heating the SWCNT network. We also identify 
a dependency on the illumination wavelength, with a slow and a fast regime of the tuning of the dielectric constant. We suppose the effect to be related to free carrier generation and dipole polarization of the nanotubes. The findings here are in agreement with our previously reported measurements of the dielectric constant of SWCNTs in the terahertz frequency range. ${ }^{23}$ The optical tuning of the dielectric constant creates novel approaches to design a wide range of optoelectronic devices such as terahertz phase shifters, frequency-selective surfaces, and metamaterials.

\section{Conflicts of interest}

There are no conflicts to declare.

\section{Acknowledgements}

We thank Dr Alexander Savin, Department of Applied Physics, Aalto University, for the help and consultation with Raman measurements. This work was financially supported in part by the European Union's (EU) Horizon 2020 Innovative Training Network CELTA (grant No. 675683 of Call: H2020-MSCA-ITN2015), by the European Research Council (ERC) under the European Union's (EU) Horizon 2020 research and innovation programme (grant No. 616846), by the Government of the Russian Federation through the ITMO Fellowship and Professorship Program, and by the Foundation for Polish Science through grants TEAM/2016-3/25 and MAB/2018/9 under the Center for Terahertz Research and Applications (CENTERA) project, carried out within the 'International Research Agendas' programme co-financed by the European Union under the European Regional Development Fund.

\section{References}

1 P. Avouris, M. Freitag and V. Perebeinos, Nat. Photonics, 2008, 2, 341-350.

2 D. Jariwala, V. K. Sangwan, L. J. Lauhon, T. J. Marks and M. C. Hersam, Chem. Soc. Rev., 2013, 42, 2824-2860.

3 A. J. Clancy, M. K. Bayazit, S. A. Hodge, N. T. Skipper, C. A. Howard and M. S. P. Shaffer, Chem. Rev., 2018, 118, 7363-7408.

4 Q. Zhang, N. Wei, P. Laiho and E. I. Kauppinen, Top. Curr. Chem., 2017, 375, 90.

5 J. C. Charlier, X. Blase and S. Roche, Rev. Mod. Phys., 2007, 79, 677-732.

6 S. Nanot, E. H. Hároz, J.-H. Kim, R. H. Hauge and J. Kono, Adv. Mater., 2012, 24, 4977-4994.

7 H. Kataura, Y. Kumazawa, Y. Maniwa, I. Umezu, S. Suzuki, Y. Ohtsuka and Y. Achiba, Synth. Met., 1999, 103, 2555-2558. 8 T. Grace, L. P. Yu, C. Gibson, D. Tune, H. Alturaif, Z. Al Othman and J. Shapter, Nanomaterials, 2016, 6, 52.

9 K. Aitola, K. Sveinbjornsson, J. P. Correa-Baena, A. Kaskela, A. Abate, Y. Tian, E. M. J. Johansson, M. Gratzel, E. I. Kauppinen, A. Hagfeldt and G. Boschloo, Energy Environ. Sci., 2016, 9, 461-466.
10 M. J. O'Connell, S. M. Bachilo, C. B. Huffman, V. C. Moore, M. S. Strano, E. H. Haroz, K. L. Rialon, P. J. Boul, W. H. Noon, C. Kittrell, J. P. Ma, R. H. Hauge, R. B. Weisman and R. E. Smalley, Science, 2002, 297, 593596.

11 F. Wang, G. Dukovic, L. E. Brus and T. F. Heinz, Phys. Rev. Lett., 2004, 92, 177401.

12 S. Tatsuura, M. Furuki, Y. Sato, I. Iwasa, M. Q. Tian and H. Mitsu, Adv. Mater., 2003, 15, 534-537.

13 A. E. Nikolaenko, N. Papasimakis, A. Chipouline, F. De Angelis, E. Di Fabrizio and N. I. Zheludev, Opt. Express, 2012, 20, 6068-6079.

14 L. Marty, E. Adam, L. Albert, R. Doyon, D. Menard and R. Martel, Phys. Rev. Lett., 2006, 96, 136803.

15 M. Steiner, M. Freitag, V. Perebeinos, A. Naumov, J. P. Small, A. A. Bol and P. Avouris, Nano Lett., 2009, 9, 3477-3481.

16 Y. Miyauchi, Z. Zhang, M. Takekoshi, Y. Tomio, H. Suzuura, V. Perebeinos, V. V. Deshpande, C. Lu, S. Berciaud, P. Kim and Others, Phys. Rev. B Condens. Matter, 2015, 92, 205407.

17 R. J. Chen, N. R. Franklin, J. Kong, J. Cao, T. W. Tombler, Y. G. Zhang and H. J. Dai, Appl. Phys. Lett., 2001, 79, 22582260.

18 E. S. Snow and F. K. Perkins, Nano Lett., 2005, 5, 2414-2417. 19 D. Suzuki, S. Oda and Y. Kawano, Nat. Photonics, 2016, 10, 809.

20 A. J. Blanch, C. E. Lenehan and J. S. Quinton, J. Phys. Chem. $B, 2010$, 114, 9805-9811.

21 A. J. Blanch, C. E. Lenehan and J. S. Quinton, Carbon, 2011, 49, 5213-5228.

22 I. V. Anoshkin, I. I. Nefedova, D. V. Lioubtchenko, I. S. Nefedov and A. V. Räisänen, Carbon, 2017, 116, 547-552.

23 S. Smirnov, I. V. Anoshkin, P. Demchenko, D. Gomon, D. V. Lioubtchenko, M. Khodzitsky and J. Oberhammer, Nanoscale, 2018, 10, 12291-12296.

24 A. A. Generalov, I. V. Anoshkin, M. Erdmanis, D. V. Lioubtchenko, V. Ovchinnikov, A. G. Nasibulin and A. V. Räisänen, Nanotechnology, 2015, 26, 045201.

25 J. L. Blackburn, T. M. Barnes, M. C. Beard, Y. H. Kim, R. C. Tenent, T. J. McDonald, B. To, T. J. Coutts and M. J. Heben, ACS Nano, 2008, 2, 1266-1274.

26 T. Hertel, R. Fasel and G. Moos, Appl. Phys. Mater. Sci. Process, 2002, 75, 449-465.

27 J. S. Lauret, C. Voisin, G. Cassabois, C. Delalande, P. Roussignol, O. Jost and L. Capes, Phys. Rev. Lett., 2003, 90, 057404.

28 X. L. Xu, K. Chuang, R. J. Nicholas, M. B. Johnston and L. M. Herz, J. Phys. Chem. C, 2009, 113, 18106-18109.

29 J. G. Wang, M. W. Graham, Y. Z. Ma, G. R. Fleming and R. A. Kaindl, Phys. Rev. Lett., 2010, 104, 177401.

30 T. Kampfrath, K. von Volkmann, C. M. Aguirre, P. Desjardins, R. Martel, M. Krenz, C. Frischkorn, M. Wolf and L. Perfetti, Phys. Rev. Lett., 2008, 101, 267403.

31 L. Perfetti, T. Kampfrath, F. Schapper, A. Hagen, T. Hertel, C. M. Aguirre, P. Desjardins, R. Martel, C. Frischkorn and M. Wolf, Phys. Rev. Lett., 2006, 96, 027401.

32 M. Freitag, Y. Martin, J. A. Misewich, R. Martel and P. H. Avouris, Nano Lett., 2003, 3, 1067-1071. 
33 N. M. Gabor, Z. H. Zhong, K. Bosnick, J. Park and P. L. McEuen, Science, 2009, 325, 1367-1371.

34 S. X. Lu and B. Panchapakesan, Nanotechnology, 2006, 17, 1843-1850.

35 A. Fujiwara, Y. Matsuoka, H. Suematsu, N. Ogawa, K. Miyano, H. Kataura, Y. Maniwa, S. Suzuki and Y. Achiba, Jpn. J. Appl. Phys., Part 2, 2001, 40, L1229-L1231.

36 J. L. Zhu, G. W. Zhang, J. Q. Wei and J. L. Sun, Appl. Phys. Lett., 2012, 101, 123117.

37 I. V. Anoshkin, A. G. Nasibulin, Y. Tian, B. Liu, H. Jiang and E. I. Kauppinen, Carbon, 2014, 78, 130-136.

38 A. Kaskela, A. G. Nasibulin, M. Y. Timmermans, B. Aitchison, A. Papadimitratos, Y. Tian, Z. Zhu, H. Jiang, D. P. Brown, A. Zakhidov and E. I. Kauppinen, Nano Lett., 2010, 10, 4349-4355.

39 Y. Tian, M. Y. Timmermans, M. Partanen, A. G. Nasibulin, H. Jiang, Z. Zhu and E. I. Kauppinen, Carbon, 2011, 49, 4636-4643.

40 A. D. McNaught and A. Wilkinson, Compendium of Chemical Terminology, Blackwell Science, 1997.

41 M. E. Itkis, D. E. Perea, S. Niyogi, S. M. Rickard, M. A. Hamon, H. Hu, B. Zhao and R. C. Haddon, Nano Lett., 2003, 3, 309-314.

42 M. E. Itkis, D. E. Perea, R. Jung, S. Niyogi and R. C. Haddon, J. Am. Chem. Soc., 2005, 127, 3439-3448.

43 Y. Tian, H. Jiang, J. von Pfaler, Z. Zhu, A. G. Nasibulin, T. Nikitin, B. Aitchison, L. Khriachtchev, D. P. Brown and E. I. Kauppinen, J. Phys. Chem. Lett., 2010, 1, 1143-1148.

44 R. B. Weisman and S. M. Bachilo, Nano Lett., 2003, 3, 12351238.
45 N. Nair, M. L. Usrey, W.-J. Kim, R. D. Braatz and M. S. Strano, Anal. Chem., 2006, 78, 7689-7696.

46 P. R. Mudimela, K. Grigoras, I. V. Anoshkin, A. Varpula, V. Ermolov, A. S. Anisimov, A. G. Nasibulin, S. Novikov and E. I. Kauppinen, J. Sens., 2012, 2012, 7.

47 S. A. Jensen, R. Ulbricht, A. Narita, X. L. Feng, K. Mullen, T. Hertel, D. Turchinovich and M. Bonn, Nano Lett., 2013, 13, 5925-5930.

48 M. C. Beard, J. L. Blackburn and M. J. Heben, Nano Lett., 2008, 8, 4238-4242.

49 S. Gong, Z. H. Zhu and Z. Li, Phys. Chem. Chem. Phys., 2017, 19, 5113-5120.

50 D. Hecht, L. B. Hu and G. Gruner, Appl. Phys. Lett., 2006, 89, 133112.

51 V. Parkash and A. K. Goel, Nanoscale Res. Lett., 2010, 5, 14241430.

52 M. He, E. Rikkinen, Z. Zhu, Y. Tian, A. S. Anisimov, H. Jiang, A. G. Nasibulin, E. I. Kauppinen, M. Niemelä and A. O. I. Krause, J. Phys. Chem. C, 2010, 114, 13540-13545.

53 Y. Y. Zhang, H. Son, J. Zhang, J. Kong and Z. F. Liu, J. Phys. Chem. C, 2007, 111, 1988-1992.

54 M. Mases, M. Noel, M. Dossot, E. McRae and A. V. Soldatov, Phys. Status Solidi B, 2011, 248, 2540-2543.

55 A. D. Avery, B. H. Zhou, J. Lee, E. S. Lee, E. M. Miller, R. Ihly, D. Wesenberg, K. S. Mistry, S. L. Guillot, B. L. Zink, Y. H. Kim, J. L. Blackburn and A. J. Ferguson, Nat. Energy, 2016, 1, 16033.

56 R. Jacquemin, S. Kazaoui, D. Yu, A. Hassanien, N. Minami, H. Kataura and Y. Achiba, Synth. Met., 2000, 115, 283-287. 